\title{
DISEÑO DE PRODUCTO TURÍSTICO EN COMUNIDAD RURAL: CASO MICRORREGION MASIACA.
}

\section{TOURIST PRODUCT DESIGN IN RURAL COMMUNITY: CASE MICRORREGION MASIACA.}

\author{
Carolina María Ayala Yanajara ${ }^{1}$, Lizette Marcela Moncayo Rodríguez ${ }^{2}$, \\ John Sosa Covarrubia ${ }^{3}$
}

\begin{abstract}
${ }^{1}$ Estudiante del octavo semestre de la Licenciatura en Administración de Empresas Turísticas del Instituto Tecnológico de Sonora, Unidad Navojoa, Ramón Corona esquina con Aguascalientes S/N Colonia ITSON, Navojoa, Sonora, México. Correo electrónico: carolina.ayala@potros.itson.edu.mx. Celular: 6421075841. https://orcid.org/0000-0002-1004-8599
\end{abstract}

\begin{abstract}
${ }^{2}$ Responsable del programa educativo de la Licenciatura en Administración de Empresas Turísticas del Instituto Tecnológico de Sonora, Unidad Navojoa, Ramón Corona esquina con Aguascalientes S/N Colonia ITSON, Navojoa, Sonora, México. Correo electrónico: lizette.moncayo@itson.edu.mx. Celular: 6421169366

${ }^{3}$ Profesor-investigador de la Licenciatura en Administración de Empresas Turísticas del Instituto Tecnológico de Sonora, Unidad Navojoa, Ramón Corona esquina con Aguascalientes S/N Colonia ITSON, Navojoa, Sonora, México. Correo electrónico: john.sosa@itson.edu.mx. Celular: 6421072195
\end{abstract}

\section{DOI https://doi.org/10.46589/rdiasf.vi36.396}

Recibido 24 de junio 2021.

Aceptado 30 de septiembre 2021

Publicado 1 de noviembre 2021

\section{Resumen:}

Esta investigación tiene como propósito saber cómo beneficiará la implementación de productos turísticos en la microrregión de Masica, la cual se ubica al suroeste del Estado de Sonora cuya población residente es considerada indígena y un alto porcentaje de sus localidades son de alta marginación, sin embargo, cuenta con un patrimonio cultural altamente conservado de la etnia Yoreme-Mayo: fiestas tradicionales, danzas, música, rituales y artesanía. Actualmente se carece de información turística para dar a conocer los recursos naturales y culturales con los que se cuenta y en la búsqueda de dar solución a esta 
problemática surge la necesidad de diseñar un producto turístico en la comunidad de Masiaca, con el fin de dar a conocer las riquezas que ofrece, así como impulsar la economía del lugar con los diferentes emprendedores de la región, a través de la llegada de los turistas. Por lo que se llevará a cabo un diseño de producto turístico, donde se crea un itinerario el cual incluirá actividades y talleres que se implementan en la comunidad con el fin de obtener resultados factibles en cuestión del emprendimiento en esta comunidad rural. Este trabajo se realizó en dos fases: la primera comprendió la búsqueda, comparación y análisis de diferentes métodos para la creación de productos turísticos, mientras que la segunda fase consistió en la elaboración de producto turístico.

Palabras clave: Entorno rural, turismo comunitario, ruta, producto turístico

\begin{abstract}
The purpose of this research is to know how the implementation of tourism products in the Masica micro-region will benefit, which is located in the southwest of the State of Sonora whose resident population is considered indigenous and a high percentage of its localities are highly marginalized, however, It has a highly conserved cultural heritage of the Yoreme-Mayo ethnic group: traditional festivals, dances, music, rituals and crafts. Currently there is a lack of tourist information to publicize the natural and cultural resources that are available and in the search to solve this problem, the need arises to design a tourist product in the Masiaca community, in order to give know the riches it offers, as well as boost the economy of the place with the different entrepreneurs of the region, through the arrival of tourists. Therefore, a tourist product design will be carried out, where an itinerary is created which will include activities and workshops that are implemented in the community in order to obtain feasible results in terms of entrepreneurship in this rural community. This work was carried out in two phases: the first included the search, comparison and analysis of different methods for the creation of tourist products, while the second phase consisted of the elaboration of tourist products.
\end{abstract}

Keywords: Rural environment, community tourism, route, tourism product 


\section{Introducción}

La comunidad de Masiaca se ubica al sur de la ciudad de Navojoa a una distancia de 46.5 kilómetros de su cabecera de comunidad que es la localidad de Masiaca, Navojoa. Esta Comunidad (tipo de núcleo agrario) integra en su territorio a 15 localidades y tiene una superficie territorial de 45,071 hectáreas de las cuales 43,819 son de uso común (Registro Agrario Nacional, 2018).

Dicha comunidad se encuentra dentro de la Provincia Fisiográfica de la Llanura Costera del Pacífico, en la Subprovincia Fisiográfica de la Llanura Costera y Deltas de Sonora y Sinaloa; Su localización geográfica está entre los $26^{\circ} 45^{\prime}$ de Latitud Norte y los $109^{\circ} 13^{\prime}$ de Longitud Oeste. Por ubicarse dentro de la Planicie Costera el territorio de la comunidad se encuentra casi en su totalidad con pendientes ligeras menores al 6\%; con solo una elevación relativamente importante al norte, buena parte del territorio se encuentra en una altitud de 270 msnm (Sánchez, et. al, 2015).

Masiaca está rodeada de un rico ecosistema de tipo desértico y costero donde predomina el matorral espinoso y las cactáceas columnares, entre ellas el bosque de Pitahaya más denso del mundo. Así mismo, cuenta con un patrimonio cultural bien conservado de la etnia Yoreme-Mayo: fiestas tradicionales, danzas, música, rituales y artesanía.

El turismo en espacios rurales ha sido promovido en América Latina como una alternativa de desarrollo rural, dentro de otras muchas, debido a que es considerado un mecanismo para diversificar los ingresos de las comunidades rurales y generar empleos; además, representa una oportunidad para valorizar la cultura agropecuaria rural, entre otros beneficios (Incubadora de Empresas ITSON, 2019).

Según la Organización Mundial del Turismo (OMT, 1994), “el turismo comprende las actividades que realizan las personas durante sus viajes y estancias en lugares distintos a su entorno habitual, por un periodo de tiempo consecutivo inferior a un año, con fines de ocio, por negocios y otros".

Tapia en el año 2013 menciona que, la concepción del desarrollo rural se encuentra claramente vinculada con la tradición de un desarrollo económico modernizador; es una extensión ramificada de esa noción hacia los espacios locales denominados rurales. Tal 
situación, si bien ha traído beneficios en algunos sectores en cuanto a la calidad y el nivel de vida de los pobladores rurales.

Por lo tanto, Cardona (2012) menciona que el turismo depende en gran medida de la buena voluntad de los residentes, cuyo apoyo es básico para un correcto desarrollo del destino turístico, sostenible en el largo plazo. Por ello, es importante profundizar en el estudio de los factores (impactos económicos, infraestructuras, cultura, gestión, valores, sociedad, tipo de turismo, factor tiempo, etc.) e interrelaciones que influyen en las actitudes de los residentes.

Sancho (2018) menciona que la corriente o flujo de divisas hacia el área de destino que produce el turismo no sólo constituye una importante fuente de ingresos para aquellas empresas o personas vinculadas directamente con la actividad turística, sino que también beneficia al resto de sectores de la economía a través del denominado efecto multiplicador, como se muestra en la figura 1.

Figura 1. El efecto multiplicador

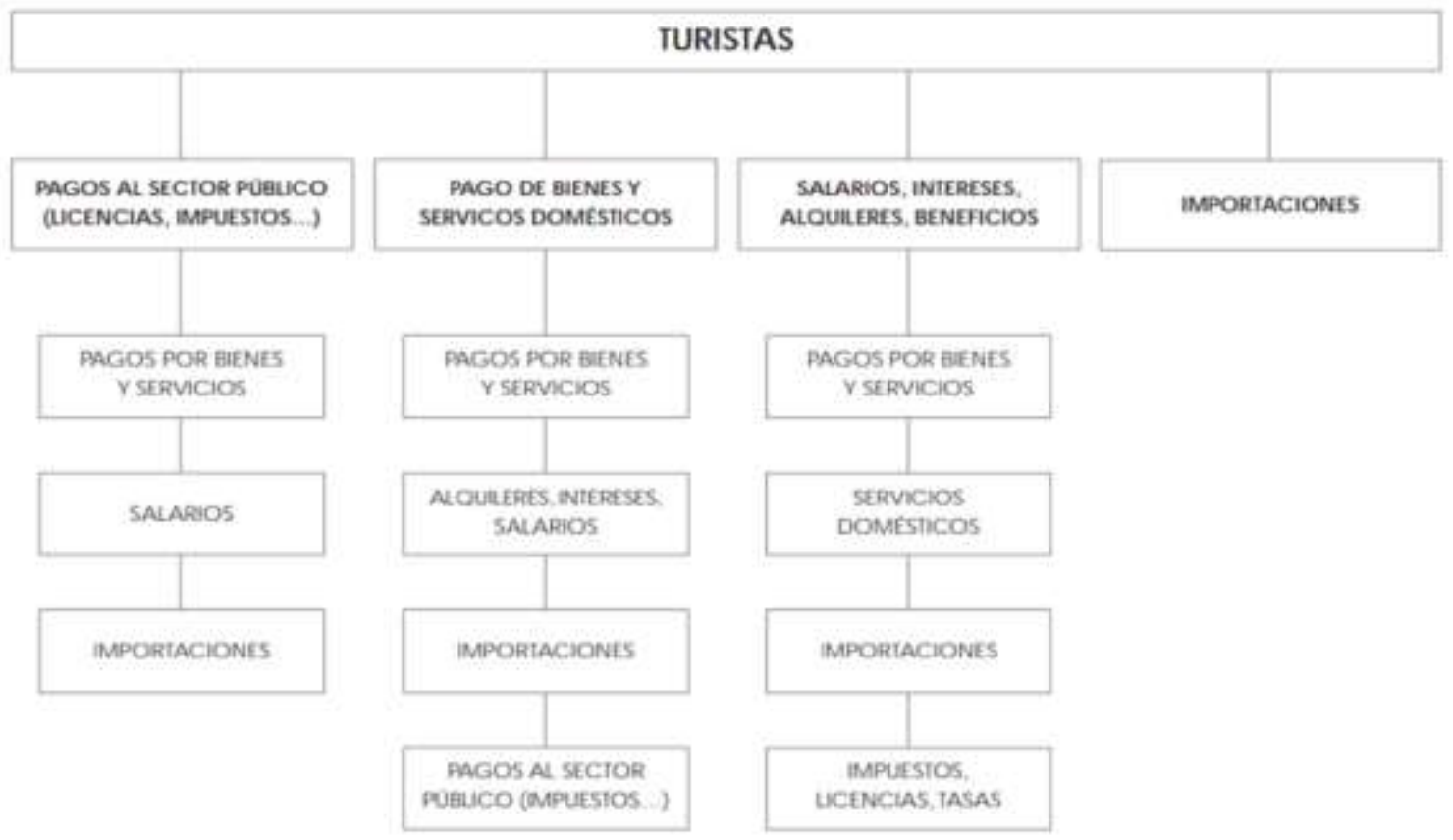

Fuente: Sancho, 2018 
En la Figura 1 se expone el funcionamiento del ya mencionado efecto multiplicador, que implica que el aumento de los ingresos por turismo genera, a su vez, un aumento en la renta de una región o país. Así, se observa como cada uno de estos flujos de renta provoca a su vez nuevos flujos, ya que los ingresos de las empresas, del sector público o de los particulares, son destinados a su vez a nuevos gastos, que conllevan un incremento de la demanda y, por consiguiente, un mayor crecimiento económico.

Así, a través de los ingresos públicos generados por el pago de impuestos y tasas por los turistas, el sector público hace frente al pago de salarios, a la compra de bienes de equipo y servicios, importaciones, etc. Asimismo, las empresas que proveen a la industria turística hacen frente, con los ingresos obtenidos por sus ventas, a pagos de mercancías y bienes procedentes de otras empresas, a pagos de otros factores de producción, importaciones, gastos de impuestos, salarios, intereses, etc.

A su vez, los salarios recibidos por los trabajadores vinculados directa o indirectamente con la actividad turística se destinan a la compra de bienes y servicios, al pago de impuestos, importaciones y también al ahorro doméstico, que beneficia a las entidades financieras y permiten el saneamiento de la economía (Sancho, 2018).

En síntesis, la actividad turística es importante ya que genera corrientes de flujos de renta que permiten un aumento del ingreso en los demás sectores de la actividad económica, redistribuyéndose la renta en un círculo cada vez mayor (Sancho, 2018).

Para Boullón (2003), el producto turístico es algo más complejo y está conformado por toda la oferta turística, integrada, a su vez, por los servicios que suministran los elementos de la planta turística y por algunos bienes no turísticos. En otras palabras, la oferta turística son todos los bienes y servicios disponibles para el turista por un precio y en un período dado, condiciones estas que determinan su característica de transitoriedad, es decir su imposibilidad de almacenamiento. Respecto a los bienes que conforman la oferta o producto turístico, a partir de lo expuesto por Boullón (2003), ningún bien puede ser considerado propiamente turístico (los bienes perecederos, de duración limitada y permanentes, la planta turística como bienes de capital) por cuanto ninguno de ellos es de consumo exclusivo del turista, sino también de consumo y distribución entre la población local; además, en el caso de la planta física, en ningún momento el turista adquiere la 
propiedad de dicho bien, sólo lo alquila. En todo caso la artesanía podría ser el único artículo que se aproximaría a ser un bien turístico.

Además Boullón (2003), resalta que los servicios no son los únicos elementos, ni los más importantes dentro del producto turístico, por el contrario, son sólo un medio que facilita la práctica del turismo; es decir, satisfacer necesidades básicas del turista (dormir, alimentarse, comunicarse o trasladarse) de cara al logro de su verdadero objetivo que no es otro que divertirse, pasear, practicar algún deporte, conocer, entre otras actividades.

De acuerdo a lo anterior, las cualidades que poseen los productos turísticos los diferencian de otros que se originan en los demás sectores económicos. Así también el producto está integrado por elementos de orden sociológico y psicológico, debido a que la creación de éstos parte de satisfacer las necesidades o deseos humanos. Por ello, Villalva (2011) enlista algunos de éstos:

- Atractivos: Son las cosas o lugares que motivan la visita del turista.

- Accesos y facilidades: Son los medios para obtener el producto turístico.

- Herencia cultural de un pueblo: Es todo aquello relacionado con la cultura de un grupo social en particular; lo cual también puede convertirse en la principal motivación para los visitantes.

La Secretaría de Turismo en México (SECTUR) en el año 2012 define al turismo rural como: los viajes que tienen como fin realizar actividades de convivencia e interacción con una comunidad rural, en todas aquellas expresiones sociales, culturales y productivas cotidianas de la misma. Actividades más reconocidas y practicadas en México para este segmento son: talleres gastronómicos, vivencias místicas, aprendizaje de dialectos, eco arqueología, preparación y uso de medicina tradicional, talleres artesanales, fotografía rural. SECTUR en el año (2011), menciona que el desarrollo económico del turismo rural ha de valorar turísticamente a) los recursos naturales, culturales y sociales con fines turísticos y b) los productos y servicios que respondan a las necesidades de esparcimiento del turista. Todo ello marcado dentro de un desarrollo local sostenido, respetuoso con el entorno y socialmente solidario con la confrontación ciudad campo donde el turismo se perfila como principal eje de desarrollo. 
Un principio importante es que turismo rural ha de armonizar los intereses del turismo, del medio ambiente y de la comunidad local. El turismo rural, como parte integrante del engranaje total del turismo, implica una actividad turística. El medioambiente y las comunidades locales son sus elementos básicos y por ello están implicados directamente en la recepción de beneficios y de costes (Bote,1990).

Una de las formas hoy más utilizadas para promover el crecimiento de una región a través del turismo es la conformación de Rutas Turísticas, las cuales consisten en unir varios sitios a través de una temática común. Se compone de un conjunto de localidades, elementos, comunidades, etc., organizados en forma de red dentro de una región determinada y que, estando debidamente señalizadas, suscitan un reconocimiento de interés turístico (Gambarota, et al, 2017).

Los operadores en turismo son aquellos agentes que participan en la actividad turística, generalmente, en calidad de intermediarios entre el consumidor final: el turista, la demanda turística, y el producto turístico: bien o servicio, destino turístico, aunque puede extender su acción intermediadora al resto de la oferta complementaria: restauración, hotel, conjunto de la oferta de alojamiento, (Sancho, 2018).

Para SECTUR (2011) el potencial turístico debe evaluar integralmente factores determinantes como, oferta y demanda actual y esperada de visitantes, para identificar la capacidad de los productos turísticos en satisfacer gastos y preferencias de los turistas. Zimmer y Grassman (1996) mencionan dos fases para evaluarlo 1) análisis de la situación turística existente, se examina la oferta, demanda, competencia y tendencias del mercado; 2) el diagnostico, se compara los resultados y el análisis, que permite identificar los puntos fuertes y debilidades del territorio, oportunidades y riesgos, y la conveniencia del desarrollo del turismo en la zona.

El itinerario turístico según Yepes (2000), consiste en un recorrido, que se realiza con el empleo de transporte turístico con paradas establecidas en atractivos, con la finalidad de desarrollar la actividad turística de forma organizada para satisfacer al turista. Cisne y Gastal (2011) agregan que el itinerario turístico debe contener explícitamente la localización y la orientación espacial del lugar o lugares que evoca, así como la descripción detallada y orientada de los elementos que componen el paisaje natural y cultural de esos 
lugares. El itinerario debe ser enriquecido con el acompañamiento de un mapa temático. Los itinerarios turísticos son planeados a partir del establecimiento de objetivos y características del segmento al cual se proyecta. Debe ser entendido como un camino para ser recorrido. El Ministerio del Poder Popular para el Turismo (MINTUR, 2009) 1o considera un programa de viaje que incluye horas de salida y llegada, desde o hacia los destinos elegidos, por lo tanto, Hernández (2014) menciona que es primordial saber el tiempo disponible para el recorrido.

Acorde con Valdez y Ochoa (2015) Las características del turismo rural surgen al analizar las diferentes definiciones que de él se han dado. Un estudio de dichas acepciones muestra, que, entre otras, estas características lo definen por desarrollarse en el medio rural, como contra posición a los núcleos urbanos y trata de unir los intereses turísticos y medioambientales con los de la comunidad local, supone una oferta turística reducida, lo que implica la usencia de masificación y la utilización de pequeñas infraestructuras en armonía con la zona en la que se encuentran ubicadas. Es un turismo que utiliza los distintos recursos naturales, patrimoniales y culturales, propios del medio rural, pero siendo respetuosos con ellos y cuidando, ante todo, su conservación y sostenibilidad. Dicha sostenibilidad conlleva el aprovechamiento óptimo de los recursos, la integración de la población local, la preservación y mejora del entorno y todo aquello que permita un proceso duradero en contraposición al modelo o concepto de la máxima rentabilidad en el espacio y tiempo que corresponde a la clara especulación y en la mayoría de los casos al modelo convencional del turismo de sol y playa.

Supone un importante factor, de desarrollo para las economías locales, ya sea de forma individualizada o como complemento a la actividad agraria.

Es básicamente demandado por turistas cuya principal motivación es el contacto con el medio rural y el conocimiento de sus particularidades, así como la huida de la masificación. Existe, por tanto, un componente educativo en esta modalidad turística, que no se da en el turismo convencional o de masas, es por ello que surge la siguiente interrogante: ¿Qué beneficio traerá a la comunidad rural de la Microrregión Masiaca, el diseño e implementación de productos turísticos a través de rutas y eventos turísticos que 
busquen lograr la participación e integración de la comunidad local como un actor principal?

Es por ello que el objetivo de la presente investigación se enfoca en proponer y diseñar un producto turístico que integre a la comunidad local en la generación de productos, no solo como atractivo por los recursos naturales y culturales con los que cuenta, sino como un actor participativo que proponga, desarrolle y lleve a cabo dichos productos para un beneficio propio del a comunidad.

\section{Método}

La investigación es de tipo cualitativa ya que utiliza la recolección de datos sin medición numérica para descubrir o afinar preguntas de investigación en el proceso de interpretación (Hernández, 2006).

La metodología a utilizar será la propuesta por Machado y Hernández (2007), la cual propone los siguientes elementos: objetivos, estudio de la oferta, desarrollo del producto, estrategias de marketing, prueba de mercado, lanzamiento del producto, seguimiento, sensibilización de la comunidad local, mapeo de actores y forma de participación de la comunidad local. Los participantes como público receptor son los integrantes de la comunidad de la Microrregión Masiaca, personas de sexo masculino y femenino con edades de entre 20 y 55 años, residentes de la microrregión, la mayoría de ellos cuentan con un nivel de estudios académicos básicos, como público emisor, colaboraron estudiantes y profesores de la Licenciatura en Administración de empresas Turísticas ITSON Navojoa, e integrantes asesores de la Incubadora de Empresas ITSON Navojoa.

\section{Resultados y discusión}

A continuación, se presentan los resultados obtenidos del diseño de un producto turístico inmerso en una ruta turística de la microrregión Masiaca para el impulso del turismo rural y del emprendimiento de negocios turísticos como se muestra en la tabla 1.

El diseño de este producto turístico formó parte del programa del evento: “Turismo rural Masiaca, explora nuevos destinos (2019)”. 
Tabla 1

Itinerario ruta turística Masiaca

\begin{tabular}{|c|c|c|}
\hline \multicolumn{3}{|c|}{ ITINERARIO } \\
\hline Hora & Descripción de actividad & Responsable \\
\hline $7: 00 \mathrm{am}$ & $\begin{array}{l}\text { Salida de ITSON con destino a } \\
\text { la comunidad de Masiaca }\end{array}$ & $\begin{array}{l}\text { Equipo colaborador } \\
\text { ITSON Navojoa }\end{array}$ \\
\hline $7: 40 \mathrm{am}$ & $\begin{array}{l}\text { Llegada a Masiaca y bienvenida } \\
\text { al grupo por parte de integrantes } \\
\text { de la comunidad en la lengua } \\
\text { Mayo y traducción al español. }\end{array}$ & $\begin{array}{l}\text { Representantes de } \\
\text { comunidad }\end{array}$ \\
\hline 8:00- 8:30am & Dinámica de integración & $\begin{array}{l}\text { Equipo colaborador } \\
\text { ITSON Navojoa }\end{array}$ \\
\hline 8:30-9:00am & Desayuno Ranchero & $\begin{array}{c}\text { Representantes de } \\
\text { comunidad }\end{array}$ \\
\hline 9:00 am- 10:00am & $\begin{array}{c}\text { Taller de tostado de café } \\
\text { Taller de elaboración de tortillas } \\
\text { de tortillas. }\end{array}$ & $\begin{array}{l}\text { Representantes de } \\
\text { comunidad }\end{array}$ \\
\hline 10:00 am- 11:00am & Juegos tradiciones & $\begin{array}{l}\text { Equipo colaborador } \\
\text { ITSON Navojoa }\end{array}$ \\
\hline 11:00am-11:30am & Visita a la piedrabola & $\begin{array}{c}\text { Equipo colaborador } \\
\text { ITSON Navojoa y } \\
\text { Representantes de } \\
\text { comunidad }\end{array}$ \\
\hline 11:30am-12:30pm & $\begin{array}{c}\text { Taller con las tejedoras" de la } \\
\text { comunidad }\end{array}$ & $\begin{array}{l}\text { Representantes de } \\
\text { comunidad }\end{array}$ \\
\hline 12:30pm- 1:00pm & Visita al aguaje & $\begin{array}{c}\text { Equipo colaborador } \\
\text { ITSON Navojoa }\end{array}$ \\
\hline $1: 00 \mathrm{pm}-1: 30 \mathrm{pm}$ & Comida tradicional & $\begin{array}{l}\text { Representantes de } \\
\text { comunidad }\end{array}$ \\
\hline 1:30pm-2:00pm & $\begin{array}{l}\text { Despedida de los anfitriones de } \\
\text { la comunidad y tiempo para que } \\
\text { el grupo vea las artesanías } \\
\text { denominadas "Benjamín" }\end{array}$ & $\begin{array}{l}\text { Representantes de } \\
\text { comunidad }\end{array}$ \\
\hline $2: 00 \mathrm{pm}-2: 30 \mathrm{pm}$ & Legada a ITSON Navojoa & $\begin{array}{l}\text { Equipo colaborador } \\
\text { ITSON Navojoa }\end{array}$ \\
\hline
\end{tabular}

Fuente: elaboración propia (2019). 
Como resultado del diseño e implementación de un producto turístico se tuvo la participación de un grupo de 34 estudiantes de la Licenciatura en Administración de Empresas Turísticas del Instituto Tecnológico de Sonora (ITSON) unidad Navojoa, así como responsables de la incubadora de empresas ITSON en el evento denominado "Turismo rural Masiaca, explora nuevos destinos (2019)".

Cabe mencionar que la invitación se hizo a través de las redes sociales oficiales del programa educativo LAET, de la incubadora de empresas ITSON y de todos los colaboradores del proyecto.

Es necesario destacar la importancia de que los habitantes de la microrregión estén preparados tanto en lo práctico como en lo teórico, pero en este caso al tratarse de personas de rancherías o comunidades cercanas, no contaban con los conocimientos suficientes, y por ello no estaban familiarizados con temas relativos al turismo, y como resultado, la interpretación y los servicios frente a los grupos se veían limitados. En este sentido, el beneficio que traerá implementar y promocionar los productos ofrecidos en la comunidad, se verá reflejado tanto para los que ofrecen servicios turísticos como para quienes lo reciben, es decir, habrá una mejoría directa para los prestadores de servicios y para los turistas.

El beneficio de practicar lo que es turismo rural comunitario, busca hacer crecer los mercados rurales, atraer demanda externa, buscando aumentar la producción fuera del ámbito local, así como cambiar los aspectos estructurales y culturales del mercado laboral; aumentando las capacidades de la población para el empleo y mejorar las infraestructuras para la actividad emprendedora.

Tal es el caso que en el año 2018 a través del Instituto Tecnológico de Sonora, Unidad Navojoa, llevó a cabo el diseño de un Plan de Desarrollo Turístico Local para el territorio de la Comunidad Indígena de Masiaca, con el objetivo de impulsar las actividades económicas, sociales y ambientales de manera integral, que involucre a la comunidad así como el aprovechamiento y la preservación de los recursos naturales, en alianzas estratégicas con los gobiernos Municipal, Estatal y Federal e instituciones públicas y privadas, elaboración de una ruta de turismo rural, capacitación en temas especializados en 
turismo, formalización de un grupo social o figura jurídica aplicable para la administración de los productos turísticos y la incubación de al menos 10 microempresas.

Para ofertar servicios competitivos y de calidad, el área de incubadora de empresas de la institución, solicitó el apoyo de profesores universitarios especializados en el turismo, para colaborar en el diseño y ejecución de un programa de capacitación para los habitantes de la microrregión Masiaca, que tienen contacto con los turistas o los que incubaron alguna de las microempresas en dicha microrregión, el cual, proporcionase las herramientas claves para el mejoramiento de los servicios ofertados al turista en el marco de diseño de productos turísticos, calidad y atención al cliente, guías turísticos y manejo higiénico de los alimentos.

Por tal motivo, se diseñó un programa de capacitación orientado al mejoramiento de la calidad en el servicio, para aumentar la oferta de productos de turismo rural, con lo cual se esperaba coadyuve al incremento de conocimientos y mejora de las habilidades de los habitantes de la microrregión, en la búsqueda de la satisfacción óptima del cliente.

El proyecto, surgió de la necesidad que tenían los habitantes de la comunidad dotar de competencias y habilidades para el mejoramiento de la competitividad de los servicios, la calidad y atención al cliente, higiene en los alimentos, el manejo de grupos, así como el diseño de productos para incrementar su oferta turística, Es decir, este grupo de personas no contaban con conocimientos y habilidades en los tópicos anteriormente mencionados, ya que en ningún momento han recibido formación o capacitación al respecto.

Otro caso de éxito de manera internacional es el realizado por la Universidad Politécnica Salesiana en ecuador en el año 2019, que a través de una propuesta metodológica para la generación de productos turísticos a partir de la comunidad local logró establecer una guía general que ayude a la integración de los residentes del destino como gestores de la actividad turística, misma que a raíz de las nuevas exigencias sociales, económicas y ambientales hacen necesario el diseño de productos turísticos donde la comunidad local sea considerada como el pilar fundamental para su creación y desarrollo. Se realizó un análisis comparativo de los procedimientos para un producto turístico, los cuales deben reunir ciertos requisitos, como son: objetivos, necesidades de los clientes, generación de ideas, concepto de producto, estrategias de marketing, análisis del negocio, 
desarrollo del producto, prueba de mercado y lanzamiento del producto. Es por ello que el objetivo central de la Universidad Politécnica Salesiana en Ecuador, buscó elaborar una propuesta metodológica para el diseño de productos turísticos a partir de la participación y gestión de este actor.

\section{Conclusión}

Actualmente, la actividad turística es visualizada como un motor de desarrollo económico para las personas que habitan en los destinos. Es por ello que, de muchas maneras, se ha tratado de incluir la participación de la comunidad local en el turismo, con poco éxito (Sebele, 2010). Por ello, resulta indispensable generar un proceso de planificación integrador, donde la comunidad local sea reconocida como pilar fundamental para la creación y gestión de los productos turísticos. En este orden de ideas, tiene su origen el turismo comunitario, no como una nueva tipología o tendencia de turismo, sino como una manera de planeación y gestión de la actividad turística, basada en el paradigma de la sustentabilidad y el emprendimiento.

La labor de los prestadores de servicios en las comunidades rurales es esencial para satisfacer los deseos y necesidades de los visitantes, y con ello mejorar su experiencia de viaje, pues él es el gran protagonista del turismo receptivo, combina el calor humano con la transmisión e interpretación de la información. Para lograr esto, implica que los habitantes de la comunidad estén debidamente formados y capacitados de manera especializada.

Algo importante que se debe de mencionar, es la buena disposición, actitud, entusiasmo y participación activa que la microrregión Masiaca mostró en el diseño de actividades, elementos fundamentales que determinaron el éxito al trabajar en su entorno rural.

Es por ello que se puede responder afirmativamente al cuestionamiento planteado en el marco introductorio, que la implementación de productos turísticos a través de rutas y eventos turísticos logran la participación e integración de la comunidad local como un actor principal basándose en el emprendimiento. 
Como aspecto fundamental en el programa, la participación activa de los integrantes y la buena disposición, actitud, entusiasmo mostrada a lo largo del programa, destacan como elementos claves que determinaron el éxito.

Se recomienda que los prestadores de servicios de la microrregión gestionen la posibilidad de acceder constantemente a cursos de capacitación y actualización a través de la comisión de turismo de la microrregión Masiaca, tanto en cuestiones técnicas de interpretación, como en estrategias para mejorar la prestación de los servicios, ya que los cambios constantes en el comportamiento de la demanda exigen cada vez más una oferta de productos y servicios turísticos de calidad.

Se recomienda por parte de la academia, que el programa de Licenciado en Administración de empresas Turísticas, pueda realizar convenios institucionales y proponer programas a través de prácticas profesionales, o incluir en el contenido de materias como guía de turistas, patrimonio natural y cultural, seminario de investigación entre otras, que puedan coadyuvar a la realización de este tipo de diseños de productos turísticos, itinerarios, rutas turísticas y programas y planes de negocios, que a través de metodologías basadas en el emprendimiento local de las comunidades, apoyen en su economía y desarrollo profesional al ofrecer un producto o prestar un servicio.

Se recomienda finalmente, diseñar y aplicar un instrumento de evaluación en función de los elementos y competencias más importantes de cada uno de los cursos impartidos.

\section{Referencias bibliográficas}

Bote, V. (1990). Planificación Económica Del Turismo. México: Trillas.

Boullón, R. (2003). Planificación del espacio Turístico. 3ra.ed. Editorial Trillas. México.

Cardona, J. R. (abril de 2012). ACTITUDES DE LOS RESIDENTES HACIA EL TURISMO EN DESTINOS TURÍSTICOS CONSOLIDADOS: EL CASO DE IBIZA. Tesis Doctorial. Palma, Islas Baleares, España.

Cisne, R., \& Gastal, S. (2011). Nueva visión sobre los itinerarios turísticos. Una contribución a partir de la complejidad. Estudios y Perspectivas en Turismo. Disponible en: https://www.redalyc.org/articulo.oa?id=180722700012. 
Gambarota, D, \& Lorda, M. (2017). el turismo como estrategia de desarrollo local. revista geográfica Venezolana, 58(2),346-359. Consultado el 05 de abril de 2021 en: Https://Www.Redalyc.Org/Articulo.Oa?Id=3477/347753793006

Hernández, M. (2014). ¿Cómo Se Elabora Un Recorrido Turístico?.

Hernández., (2006). Metodología de la investigación. Mc Graw Hill 4ta. Edicion.

Incubadora de Empresas ITSON, 2019. Informe Masiaca.

MACHADO, E., \& HERNÁNDEZ, Y. 2007. Procedimiento para el diseño de un producto turístico integrado en Cuba. Teoría y Praxis(4), 161-174.

MINTUR (Ministerio de Turismo del Ecuador). 2007. Inventario de Atractivos

OMT, (1994): Les GDSs dans l'Industrie Touristique, Madrid.

Registro Agrario Nacional (RAN), (2018). Padrón e Historial de Núcleos Agrarios (PHINA). Recuperado de: http://www.ran.gob.mx/ran/index.php/sistemas-deconsulta/phina.

Sánchez, et. al, 2015 TÉCNICA DE BOLSEO: SISTEMA DE CAPTACIÓN DE AGUA PARA SIEMBRAS EN HUMEDAD RESIDUAL UTILIZADO POR PRODUCTORES DE MASIACA, SONORA. Número 5 Edición Especial Universidad Autónoma Indígena de México Mochicahui, El Fuerte, Sinaloa. pp. $139-155$

Sancho, A. (2018), Introducción Al Turismo. Madrid: Organización Mundial De Turismo.

Sebele, L. 2010. Community-based tourism ventures, benefits and challenges: khama rhino sanctuary trust, central district, botswana. Tourism management, 31(1), 136-146. Consultado el 05 de abril de 2021 en https://doi.org/10.1016/j.tourman.2009.01.005.

SECTUR. (2011). Identificación De Potencialidades Turísticas En Regiones Municipales.

Tapia, F. H. (2013). Enfoques y políticas de desarrollo rural en México: Una revisión de su construcción institucional. SciELO, 131-159.

Turísticos del Cantón Pedernales. Portoviejo.

Valdez, P. Ochoa, S. (2015), Instituto Tecnológico De Sonora. Consultado el 6 de abril de 2021 en: https://www.itson.mx/publicaciones/documents/cienciaseconomico/turismorural.pdf. 
VILLALVA, D. 2011. Diseños de productos turísticos sostenibles para el Cantón Cumandá provincia de Chimborazo. Tesis de ingenieria en Ecoturismo. Escuela Superior Politécnica de Chimborazo: Facultad de Recursos Naturales. Ecuador.

Yepes, V. (2000). Los itinerarios temáticos como elementos diferenciadores del producto turístico global en: http://personales.upv.es/vyepesp/Presentaciones/itinerarios.pdf Zimmer, P., Grassmann S. (1996). Evaluar el potencial turístico de un territorio. Leador European observatory.

\section{Cómo citar:}

Ayala Yanajara, C. ., Moncayo Rodríguez, L., \& Sosa Covarrubias, J. . (2021). Diseño de producto turístico en comunidad rural: caso microrregion Masiaca. Revista De Investigación Académica Sin Frontera: División De Ciencias Económicas Y Sociales, (36). https://doi.org/10.46589/rdiasf.vi36.396

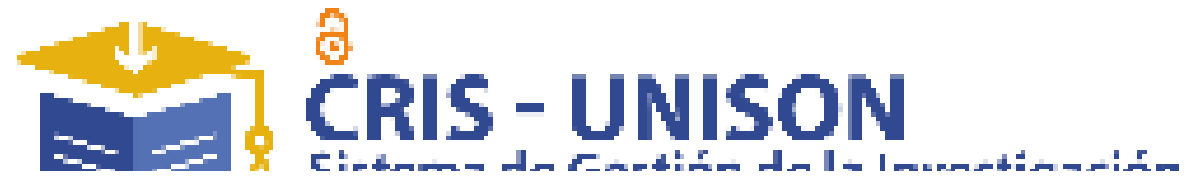

- Dial net
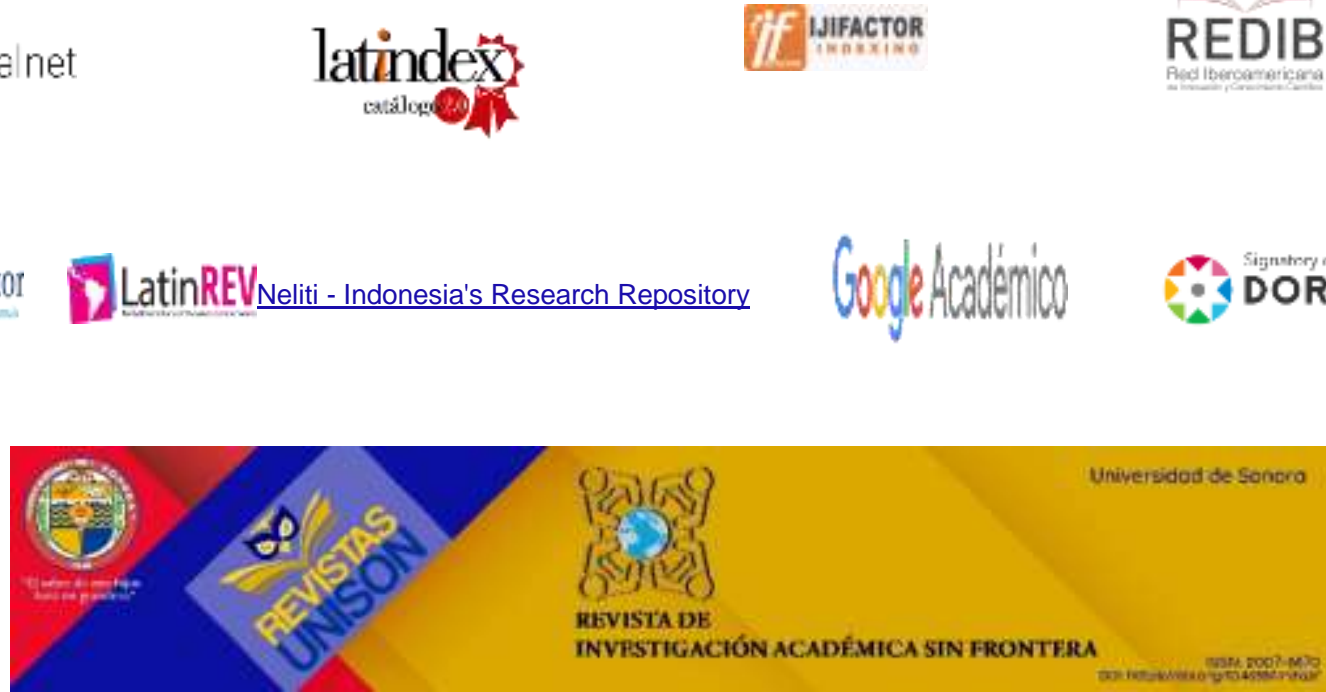
Año 14 / Núm. 36 / julio-diciembre 2021 